\title{
Benefits of Nifedipine GITS in Stable Coronary Artery Disease: Further Analysis of the "ACTION" Database
}

Peter A. Meredith · Henry L. Elliott

Received: April 9, 2010 / Published online: May 18, 2010

(C) The Author(s) 2010. This article is published with open access at Springerlink.com

\section{ABSTRACT}

Introduction: Retrospective analyses of specific subgroups of patients from the database of the ACTION study have evaluated the effectiveness of a nifedipine gastrointestinal therapeutic system (GITS) on clinical outcomes. These subgroups included those patients receiving: 1) full "optimal" therapy at baseline; 2) full "optimal" therapy at baseline but excluding renin angiotensin system (RAS)-blocking drugs; 3) treatment with nifedipine GITS who were not treated with RAS blockers versus those treated with RAS blockers but not nifedipine GITS. Methods: Analyses were performed on an intention-to-treat basis. Treatment groups were compared by log-rank test without adjustment for covariates. Hazard ratios with 95\% confidence intervals were obtained using Cox proportional

Peter A. Meredith $(\bowtie)$

Medicine \& Therapeutics, University of Glasgow,

Western Infirmary, Glasgow, UK.

Email:pam1v@clinmed.gla.ac.uk

Henry L. Elliott

Institute of Pharmaceutical \& Biomedical Sciences,

University of Strathclyde, Glasgow, UK hazards models with treatment allocation as the only covariate. Results: 2461 patients randomized in ACTION were receiving optimal therapy (beta blockers, nitrates, aspirin, statins) excluding RAS blockers at baseline. There were reductions associated with nifedipine GITS compared with placebo in all prespecified endpoints but statistical significance was only achieved for debilitating stroke $(48 \% ; P<0.02)$ and coronary angiography (14\%; $P<0.05)$. These benefits were paralleled by a -4.1 and $-2.8 \mathrm{mmHg}$ difference between the groups for systolic and diastolic blood pressure, respectively. Patients randomized to nifedipine GITS but no RAS blockers $(n=2966)$ when compared to those receiving RAS blockers but no nifedipine GITS ( $n=880$ ) had highly statistically significant reductions in cardiovascular events (22\%), newonset heart failure (53\%), and debilitating stroke (45\%). However, the groups differed in their baseline characteristics. Conclusion: Addition of nifedipine GITS to the treatment regimen of selected patient groups with symptomatic coronary artery disease results in a significant reduction of cardiovascular morbidity. While the interpretation of these subgroup analyses must obviously be cautious, there is a clear message relating to "best practice" treatment of angina, 
which suggests that "reliance" on RAS blockade may be misplaced and greater attention should be directed towards control of blood pressure.

Keywords: ACE inhibitor; angina pectoris; angiotensin receptor blockers; blood pressure; calcium channel blockers; combination drug therapy; coronary artery disease; hypertension; nifedipine

\section{INTRODUCTION}

The European guidelines for the treatment of stable angina pectoris recommend that, in addition to risk factor modification, the focus should be upon improving prognosis and minimizing symptoms. ${ }^{1}$ The recommended algorithm for improving prognosis suggests that the regimen should include anti-thrombotic drugs (usually low-dose aspirin), lipid lowering with statins, angiotensin-converting enzyme (ACE) inhibitors, and beta-blockers. Where symptoms persist, the guidelines also suggest the use of (long-acting) calcium channel blockers (CCBs). These recommendations are, in part, founded on evidence-based medicine and a logical interpretation of the available data from randomized controlled trials. However, it should be recognized that much of the guidance is based upon "established practice" and benefit by way of improvement of symptoms. There is a relative paucity of definitive outcome trials and the available evidence is not wholly consistent. For example, although the HOPE study (Heart Outcomes Prevention Evaluation) ${ }^{2}$ demonstrated that there were significant and clinically relevant benefits with ACE inhibitor treatment (ramipril) in patients with stable coronary artery disease, the magnitude of the benefit in the EUROPA trial (EURopean trial On reduction of cardiac events with Perindopril in stable coronary
Artery disease) $)^{3}$ was much smaller and there was no apparent benefit in the PEACE trial (Prevention of Events in ACE Inhibition) (with trandolapril). ${ }^{4}$ With respect to $\mathrm{CCB}$ treatment, ACTION (A Coronary disease Trial Investigating Outcome with Nifedipine gastrointestinal therapeutic system [GITS]) established that nifedipine (in its long-acting GITS formulation) can improve the prognosis of patients with chronic stable angina, particularly in those with concomitant hypertension. . $^{5,6}$

This further retrospective analysis of data derived from ACTION addresses practical treatment issues in patients with established stable coronary artery disease. With the ultimate aim of identifying optimal treatment strategies in this group of high-risk patients, the analysis specifically focuses on the role of the long-acting $\mathrm{CCB}$, nifedipine GITS.

\section{MATERIALS AND METHODS}

\section{Patients and Methods}

The design, methods, and main results of the ACTION trial have been published in detail previously. ${ }^{5,7}$ In brief, patients with angina pectoris were randomized to receive either nifedipine GITS or matching placebo. In addition to angina, patients had to have either a history of myocardial infarction, proven angiographic coronary artery disease, or a positive exercise test or perfusion defect. The left ventricular ejection fraction had to be at least $40 \%$. Selection criteria and definitions have been previously described in detail elsewhere. ${ }^{7}$ The starting dose of nifedipine GITS or placebo was $30 \mathrm{mg}$ once daily, increasing to $60 \mathrm{mg}$ once daily within 6 weeks. These treatments were in addition to "best practice" cardiovascular therapy, with a follow-up period of 4 years. This further analysis has explored a number of aspects of the 
effectiveness of different treatment options in patients with chronic stable angina.

\section{Statistical Methods}

This present analysis has focused on a number of composite endpoints that were predefined prior to the conduct of the ACTION trial. The endpoints included: the combined rate of death from any cause, myocardial infarction, refractory angina requiring coronary angiography, new overt heart failure requiring hospitalization, and peripheral revascularization (the ACTION primary endpoint for efficacy); the combined rate of death from any cause, myocardial infarction, and debilitating stroke (the ACTION primary endpoint for safety); any cardiovascular event (the ACTION primary endpoint for efficacy minus noncardiovascular death); any death, cardiovascular event, or procedure (the ACTION primary endpoint for efficacy plus coronary angiography, percutaneous coronary intervention, and coronary bypass surgery); and any vascular event or procedure (the ACTION primary endpoint for efficacy minus noncardiovascular death and new overt heart failure, plus percutaneous coronary intervention and coronary bypass surgery).

All analyses for composite outcomes and clinical events were performed on an intentionto-treat basis. Deaths of unknown cause were considered as cardiovascular. Coronary angiography and percutaneous coronary intervention on the same day were counted only as percutaneous coronary intervention. Treatment groups were compared by the log-rank test without adjustment for covariates or interim analysis. Hazard ratios with 95\% confidence intervals were obtained using Cox proportional hazards models with treatment allocation as the only covariate.
Thus, these present analyses have explored the outcome benefits attributable to the addition of nifedipine GITS in relation to the following different patterns of concurrent treatment:

1. Full "optimal" therapy at baseline (including beta blockers, nitrates, aspirin, statins, and renin angiotensin system [RAS] blockers).

2. Full "optimal" therapy at baseline (as above) but excluding RAS-blocking drugs.

3. Treatment with nifedipine GITS in patients who were not treated with RAS blockers versus treatment with RAS blockers in patients who did not receive nifedipine GITS.

\section{RESULTS}

In brief, 7655 patients with angina pectoris were randomized to receive either nifedipine GITS ( $n=3825)$ or matching placebo $(n=3840)$.

\section{1) Outcome in Patients Receiving "Optimal" Therapy at Baseline}

Evaluation of the database of 7665 patients randomized in ACTION revealed that at baseline only 464 were receiving beta blockers, nitrates, aspirin, statins, and RAS blockers-this being defined as full optimal therapy as recommended by the current European guidelines. ${ }^{1}$ Although the two groups (patients with angina pectoris receiving either nifedipine GITS or placebo) were well matched for baseline characteristics, the small number of patients precluded any meaningful statistical analysis due to the lack of statistical power. Nonetheless, the summarized outcome findings are presented in Table 1. Those patients randomized to nifedipine GITS attained lower blood pressure (BP) by the end of the trial (visit 18) than those randomized to placebo. The mean difference between the groups was -5.1 and $-2.7 \mathrm{mmHg}$ for systolic and diastolic 
Table 1. Blood pressure and outcome in patients receiving "optimal" therapy at baseline.

\begin{tabular}{lccc}
\hline & $\begin{array}{c}\text { Nifedipine } \\
(\boldsymbol{n = 2 2 4})\end{array}$ & $\begin{array}{c}\text { Placebo } \\
(\boldsymbol{n}=\mathbf{2 4 0})\end{array}$ & \\
\hline SBP/DBP at baseline $(\mathrm{mmHg})$ & $142.3 / 81.7$ & $140.9 / 81.6$ & \\
SBP/DBP at visit 18 (mmHg) & $134.7 / 76.8$ & $138.3 / 79.3$ & \\
Change in SBP/DBP (mmHg) & $-7.4 /-4.8$ & $-2.3 /-2.1$ & \\
\hline & Hazard ratio & Lower 95\% CI & Upper 95\% CI \\
\hline Primary efficacy & 0.866 & 0.588 & 1.275 \\
Primary safety & 0.834 & 0.523 & 1.330 \\
Any CV event & 0.835 & 0.558 & 1.247 \\
Death, any CV event, or revascularization & 0.982 & 0.745 & 1.296 \\
Any vascular event or revascularization & 0.863 & 0.621 & 1.199 \\
Heart failure & 0.641 & 0.252 & 1.629 \\
Debilitating stroke & 0.782 & 0.248 & 2.465 \\
Coronary angiography & 0.978 & 0.695 & 1.377 \\
Acute myocardial infarction & 0.917 & 0.499 & 1.683 \\
\hline
\end{tabular}

$\mathrm{SBP} / \mathrm{DBP}=$ systolic/diastolic blood pressure; $\mathrm{CV}=$ cardiovascular.

BP, respectively. All of the predefined outcomes favored nifedipine GITS with a $13 \%$ reduction in the primary outcome of efficacy, a $36 \%$ reduction in new-onset heart failure, and a $22 \%$ reduction in debilitating stroke (Table 1). None of the reductions achieved statistical significance.

\section{2) Outcome in Patients Receiving "Optimal" Therapy Excluding RAS Blockers at Baseline}

A total of 2461 patients randomized in ACTION were receiving optimal therapy excluding RAS blockers at baseline. The demographic and clinical features of the 1223 patients randomized to nifedipine GITS and 1238 to placebo are detailed in Table 2 along with the drug therapy at baseline. It is apparent from Table 2 that although these patients are a selected group from the randomized population they are well matched for the baseline characteristics with no statistically significant differences between the two groups. Four years after randomization (visit 18), the achieved BP in the nifedipine GITS and placebo group were 129.9/75.2 and
134.4/78.0 $\mathrm{mmHg}$, respectively. This represents $\mathrm{a}-4.1$ and $-2.8 \mathrm{mmHg}$ difference in between the groups for SBP and DBP, respectively. Figure 1 shows the effects of nifedipine GITS (relative to placebo) on predefined ACTION combined endpoints in patients receiving optimal therapy excluding RAS blockers at baseline. As evidenced by $95 \%$ confidence intervals that do not include "no effect" (hazard ratio=1), nifedipine significantly reduced debilitating stroke by $48 \%$ $(P<0.02)$ and the coronary angiography by $14 \%$ $(P<0.05)$. There also were reductions associated with nifedipine GITS in all the other prespecified endpoints, but these did not achieve statistical significance (Figure 1).

\section{3) Outcome in Patients Receiving Nifedipine GITS but no RAS Blockers, Compared to Those Receiving RAS Blockers but No Nifedipine GITS}

At total of 2966 patients in ACTION were randomized to nifedipine GITS but were not treated with RAS blockers, whilst 880 patients 
Table 2. Demographic and clinical features and drug therapy in patients receiving "optimal" therapy excluding RAS blockers at baseline.

\begin{tabular}{|c|c|c|}
\hline & $\begin{array}{c}\text { Nifedipine } \\
(n=1223)\end{array}$ & $\begin{array}{c}\text { Placebo } \\
(n=1238)\end{array}$ \\
\hline \multicolumn{3}{|l|}{ Demographics } \\
\hline Age, years $($ mean $\pm S D)$ & $62.9 \pm 9.2$ & $62.2 \pm 9.3$ \\
\hline Men, \% & 76.1 & 77.6 \\
\hline \multicolumn{3}{|l|}{ Clinical features, \% } \\
\hline History of myocardial infarction (with coronary revascularization) & $52.3(25.8)$ & $50.6(25.0)$ \\
\hline Angiographic coronary artery disease, no MI (with coronary revascularization) & $33.2(20.6)$ & $33.3(19.7)$ \\
\hline Positive exercise or radionuclide test only & 14.1 & 15.7 \\
\hline No history of coronary artery disease & 0.41 & 0.49 \\
\hline Significant lesions on coronary angiogram & 72.5 & 70.2 \\
\hline Past use of calcium antagonists & 21.8 & 21.0 \\
\hline Anginal attacks & 93.8 & 93.1 \\
\hline \multicolumn{3}{|l|}{ Risk factors, $\%$} \\
\hline Current smoker & 18.4 & 18.6 \\
\hline Total cholesterol $\geq 5.0 \mathrm{mmol} / \mathrm{L}$ & 55.3 & 57.5 \\
\hline Body mass index $\geq 30.0 \mathrm{~kg} / \mathrm{m}^{2}$ & 23.5 & 24.3 \\
\hline Diabetes mellitus & 13.8 & 11.5 \\
\hline \multicolumn{3}{|l|}{ Cardiovascular variables, mean \pm SD } \\
\hline Heart rate, b.p.m. & $62.6 \pm 9.8$ & $62.5 \pm 9.5$ \\
\hline $\mathrm{SDB}, \mathrm{mmHg}$ & $135.9 \pm 18.6$ & $135.9 \pm 18.6$ \\
\hline DBP, $\mathrm{mmHg}$ & $79.1 \pm 9.4$ & $79.1 \pm 9.7$ \\
\hline \multicolumn{3}{|l|}{ Antianginal drug, \% } \\
\hline Beta blocker & 10 & 100 \\
\hline Organic nitrate, as needed & 74.9 & 73.4 \\
\hline Organic nitrate, daily maintenance & 46.6 & 46.2 \\
\hline Other vasodilator & 2.9 & 3.2 \\
\hline \multicolumn{3}{|l|}{ Lipid-lowering drug, \% } \\
\hline Statin & 100 & 100 \\
\hline Fibrate & 1.9 & 2.1 \\
\hline Other & 1.4 & 1.9 \\
\hline Any of the above & 100 & 100 \\
\hline \multicolumn{3}{|l|}{ BP-lowering drug, $\%$} \\
\hline ACE inhibitor & 0 & 0 \\
\hline Angiotensin-II antagonist & 0 & 0 \\
\hline Diuretic & 10.6 & 9.5 \\
\hline Other & 2.5 & 2.1 \\
\hline \multicolumn{3}{|l|}{ Other cardiovascular drug, $\%$} \\
\hline Acetylsalicylic acid & 100 & 100 \\
\hline Vitamin $\mathrm{K}$ antagonist & 0.7 & 0.7 \\
\hline
\end{tabular}

$\mathrm{ACE}=$ angiotensin-converting enzyme; $\mathrm{MI}=$ myocardial infarction; $\mathrm{RAS}=$ renin angiotensin system; $\mathrm{SBP} / \mathrm{DBP}=$ systolic/diastolic blood pressure. 
Figure 1. Prespecified cardiovascular outcomes in patients receiving "optimal” therapy excluding RAS blockade at baseline. $\mathrm{CV}=$ cardiovascular; $\mathrm{HR}=$ hazard ratio; $\mathrm{NS}=$ nonsignificant.

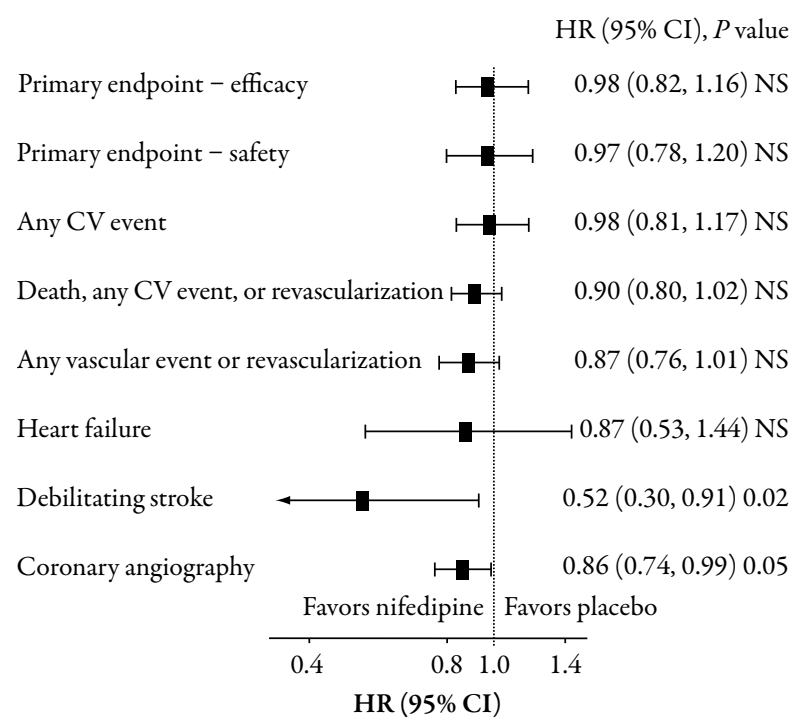

were treated with RAS blockers but did not receive nifedipine GITS (placebo). The demographic and clinical features of these patients are detailed in Table 3 along with the drug therapy at baseline. It is apparent from Table 3 that these groups differed in their baseline characteristics. The use of RAS blockade was statistically more likely in patients with a previous myocardial infarction and diabetes mellitus. These patients also had markedly higher baseline BP at $143.1 \pm 18.9 / 82.1 \pm 9.4 \mathrm{mmHg}$ compared with $135.7 \pm 18.1 / 79.2 \pm 9.3$ in those patients receiving nifedipine GITS. During the course of the trial, $\mathrm{BP}$ was reduced in both groups such that 4 years after randomization (visit 18) the achieved BP in the nifedipine GITS (no RAS blockade) group was $129.7 / 75.4 \mathrm{mmHg}$ and in the placebo group (with RAS blockade) was 139.0/79.5 mmHg. This represents a -1.7 and $-1.2 \mathrm{mmHg}$ difference in BP reduction during the course of the trial between the groups for SBP and DBP, respectively. Figure 2 shows the comparison of the two groups for the predefined ACTION endpoints. There were
Figure 2. Prespecified cardiovascular outcomes in patients receiving nifedipine GITS with no RAS blockade and patients on placebo with RAS blockade. $\mathrm{CV}=$ cardiovascular; $\mathrm{HR}=$ hazard ratio; $\mathrm{NS}=$ nonsignificant.

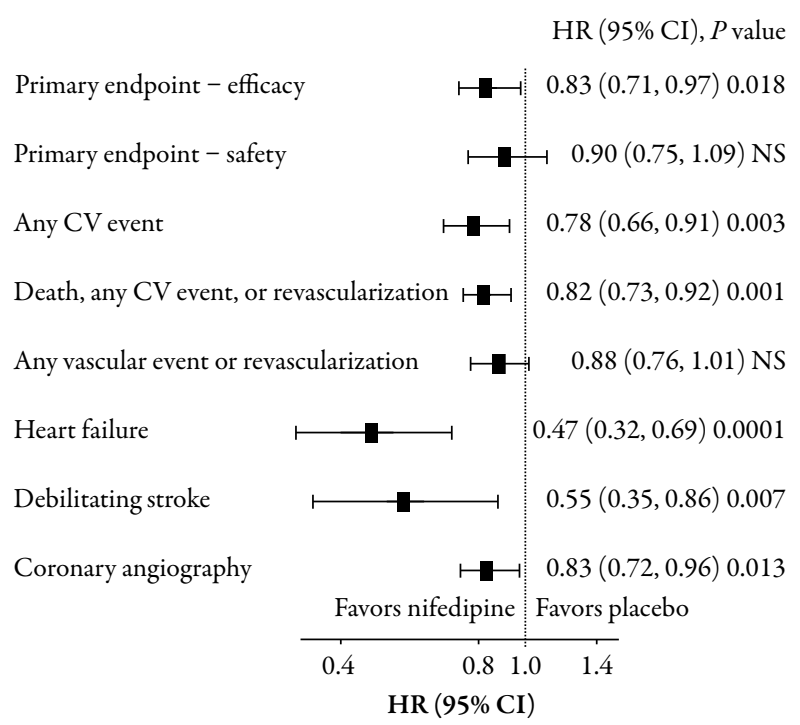

reductions associated with nifedipine GITS in all the prespecified endpoints and these achieved statistical significance in all cases except the primary endpoint for safety and any vascular event or revascularization (Figure 2). There were highly statistically significant reductions in any cardiovascular events (22\%), new-onset heart failure (53\%), and debilitating stroke (45\%).

\section{DISCUSSION}

Overall, the findings of these further analyses of the ACTION database suggest that there is considerable scope for improvement in the drug treatment of patients with stable coronary artery disease. The original aim of the ACTION trial was to establish the safety and efficacy of nifedipine GITS when added to "best practice treatment" of angina pectoris, ${ }^{5,7}$ but it is now apparent that "best practice" or "optimal" treatment reflected an algorithm for drug therapy derived from disparate levels of evidence with, in many 
Table 3. Demographic and clinical features and drug therapy in patients receiving nifedipine GITS with no RAS blockade and patients on placebo with RAS blockade.

\begin{tabular}{|c|c|c|}
\hline & $\begin{array}{l}\text { Nifedipine } \\
(n=2966)\end{array}$ & $\begin{array}{l}\text { Placebo } \\
(n=880)\end{array}$ \\
\hline \multicolumn{3}{|l|}{ Demographics } \\
\hline Age, years $($ mean $\pm S D)$ & $63.4 \pm 9.4$ & $63.7 \pm 9.2$ \\
\hline Men, \% & 80.6 & 76.5 \\
\hline \multicolumn{3}{|l|}{ Clinical features, \% } \\
\hline History of myocardial infarction (with coronary revascularization) & $50.2(23.8)$ & $57.5(29.4)$ \\
\hline Angiographic coronary artery disease, no MI (with coronary revascularization) & $32.5(20.8)$ & $30.2(17.8)$ \\
\hline Positive exercise or radionuclide test only & 17.0 & 11.9 \\
\hline No history of coronary artery disease & 0.37 & 0.34 \\
\hline Significant lesions on coronary angiogram & 67.8 & 73.1 \\
\hline Past use of calcium antagonists & 21.9 & 21.0 \\
\hline Anginal attacks & 92.7 & 91.9 \\
\hline \multicolumn{3}{|l|}{ Risk factors, \% } \\
\hline Current smoker & 18.7 & 14.2 \\
\hline Total cholesterol $\geq 5.0 \mathrm{mmol} / \mathrm{L}$ & 62.6 & 64.4 \\
\hline Body mass index $\geq 30.0 \mathrm{~kg} / \mathrm{m}^{2}$ & 20.6 & 27.0 \\
\hline Diabetes mellitus & $12.5 \%$ & $23.0 \%$ \\
\hline \multicolumn{3}{|l|}{ Cardiovascular variables, mean $\pm S D$} \\
\hline Heart rate, b.p.m. & $63.4 \pm 10.1$ & $66.7 \pm 10.9$ \\
\hline SBP, mmHg & $135.7 \pm 18.1$ & $143.1 \pm 18.9$ \\
\hline $\mathrm{DBP}, \mathrm{mmHg}$ & $79.2 \pm 9.3$ & $82.1 \pm 9.4$ \\
\hline \multicolumn{3}{|l|}{ Antianginal drug, $\%$} \\
\hline Beta blocker & 81. & 79.7 \\
\hline Organic nitrate, as needed & 58.8 & 48.8 \\
\hline Organic nitrate, daily maintenance & 37.2 & 39.6 \\
\hline Other vasodilator & 3.7 & 5.7 \\
\hline \multicolumn{3}{|l|}{ Lipid-lowering drug, \% } \\
\hline Statin & 63.3 & 63.1 \\
\hline Fibrate & 5.9 & 7.6 \\
\hline Other & 1.0 & 1.4 \\
\hline Any of the above & 68.0 & 69.2 \\
\hline \multicolumn{3}{|l|}{ BP-lowering drug, $\%$} \\
\hline ACE inhibitor & 0 & 90.0 \\
\hline Angiotensin-II antagonist & 0 & 10.6 \\
\hline Diuretic & 7.9 & 23.5 \\
\hline Other & 2.8 & 2.5 \\
\hline \multicolumn{3}{|l|}{ Other cardiovascular drugs, \% } \\
\hline Acetylsalicylic acid & 86.2 & 84.9 \\
\hline Vitamin $\mathrm{K}$ antagonist & 3.9 & 5.1 \\
\hline
\end{tabular}

$\mathrm{ACE}=$ angiotensin-converting enzyme; GITS=gastrointestinal therapeutic system; $\mathrm{MI}=$ myocardial infarction; $\mathrm{RAS}=$ renin angiotensin system; $\mathrm{SBP} / \mathrm{DBP}=$ systolic/diastolic blood pressure. 
instances, of proof of efficacy being based upon reducing symptoms rather than improving outcome. ${ }^{1}$ However, despite the relatively longestablished consensus on "optimal" treatment, only $6 \%$ of patients randomized in ACTION were receiving the full combination of beta blockers, nitrates, aspirin, statins, and drugs that block the RAS. ${ }^{7}$ Nevertheless, it might have been anticipated that the outcome benefits seen in ACTION following the addition of nifedipine GITS ${ }^{5,6}$ would predominantly have occurred in those patients receiving less than "optimal" therapy. In fact, the lowest rates for cardiovascular events were seen when treatment with nifedipine GITS was additional to "optimal" therapy (although the numbers in this retrospective analysis are too small for this to be considered definitive result). In addition to reducing cardiovascular morbidity, nifedipine treatment was associated with an expected further reduction in BP but it also should be borne in mind that nifedipine would likely also have provided additional symptomatic, antianginal benefit in such patients.

In those patients whose "optimal" therapy did not contain a RAS-blocking drug at baseline, the assessment of cardiovascular outcomes again suggested that nifedipine GITS offered specific benefits: particularly a $48 \%$ reduction in debilitating stroke and a $14 \%$ reduction in coronary angiography. Although these groups were not independently randomized, they were found to be well matched at baseline with no statistical differences in their demographic features. The outcome benefits were paralleled by a greater BP reduction in the nifedipine GITS group, but nonetheless the selective benefit by way of stroke reduction is comparable with that achieved by ramipril in the HOPE trial. ${ }^{2}$ Thus, it is reasonable to conclude that significant outcome benefits can be achieved through the addition of nifedipine GITS to the treatment regimen of patients with stable coronary artery disease who are, for whatever reason, not receiving treatment with either an ACE inhibitor or an angiotensin receptor blocker (but who are otherwise receiving "optimal" therapy).

Combination treatment with nifedipine GITS and RAS blockade was the subject of previous retrospective analyses of the ACTION database attempting to evaluate whether RAS blockade was sufficient for cardioprotection or whether or not the addition of calcium CCBs with nifedipine GITS might offer additional benefit in the subgroup of patients who were already receiving "best practice," "intensive" treatment including RAS blockade (ie, an ACE inhibitor and/or angiotensin receptor blocker). ${ }^{8}$ The results of this further analysis have identified that additional prognostic benefit was derived from the addition of nifedipine GITS. These benefits were related not only to those endpoints that have previously been shown to be predominantly associated with BP reduction, such as heart failure, ${ }^{9}$ but also to those that are BP-independent and can be attributed to the anti-ischemic properties of nifedipine (eg, coronary angiography). The overall trend was for greater reductions in all of the endpoints in the patients receiving the combination of RAS blockade and CCBs (nifedipine GITS) than for the overall ACTION population, despite a smaller differential BP reduction. ${ }^{8}$

The final further analysis in this present report was an assessment of the outcomes in those patients receiving nifedipine GITS but no RAS blockade compared with patients receiving placebo together with RAS blockade; this was specifically designed to assess the relative merits of CCBs compared to RAS blockade in patients with chronic stable angina. In this case, the analysis was confounded because there were significant discrepancies between the baseline characteristics of the two groups. 
In particular, the group of patients receiving RAS blockade can be considered to be at greater cardiovascular risk on account of higher rates for previous myocardial infarction, diabetes, and a significantly higher baseline BP. Seeking to adjust for such discrepancies in a retrospective analysis is fraught with difficulties. It is thus not possible to deduce whether or not the significant improvement in outcome is solely associated with nifedipine GITS or whether it is a function of the higher cardiovascular risk in those not receiving nifedipine. Nevertheless, even if this analysis is considered to be "observational," it is important to reflect that risk factor modulation in those patients receiving RAS blockade in the absence of nifedipine GITS is suboptimal particularly with respect to BP control.

The summarizing conclusion from all of this evidence is that the attainment of lower BP in these high-risk individuals is of paramount importance. Whilst this principle is widely acknowledged, it appears to be only slowly influencing the practice of clinical cardiologists and general practitioners. Since the publication of the HOPE trial ${ }^{2}$ in 2000, there has been a widespread perception that treatment with ACE inhibitor drugs_-and ramipril in particular-is selectively beneficial in the treatment of patients with established coronary artery disease and that these benefits are not attributable to BP lowering alone. There is also a perception that the same arguments of selective benefit beyond BP control apply in patients who suffer not only from angina but also from diabetes. This position has been sustained despite the lessconvincing findings of the EUROPA ${ }^{3}$ and PEACE ${ }^{4}$ trials. Overall, it appears that too much reliance is placed upon the selective benefits that may be offered by RAS blockade with insufficient attention being paid to the modulation of risk factors and the attainment of "tight BP control" in particular. Since the publication of $\mathrm{HOPE}^{2}$ an important additional consideration is that the "standard" therapy for coronary artery disease has become more intensive. Thus, for example, some of the differences between the results of $\mathrm{HOPE}^{2}$ and those of PEACE ${ }^{4}$ may be explained by more intensive prior drug treatment and better risk factor control in the PEACE ${ }^{4}$ population. These baseline differences obviously would reduce the likelihood of a relatively welltreated, low-risk population benefitting from additional ACE inhibitor treatment, as in the $\mathrm{PEACE}^{4}$ trial. The same interpretation can be applied to the muted effectiveness of the angiotensin receptor blocker, telmisartan, in the Telmisartan Randomised AssessmeNt Study in ACE iNtolerant subjects with cardiovascular Disease (TRANSCEND) study. ${ }^{10}$

\section{CONCLUSION}

In conclusion, the present analyses show that the addition of nifedipine GITS to the treatment regimen of selected patient groups with symptomatic coronary artery disease resulted in a significant reduction of cardiovascular morbidity. The interpretation of these analyses must obviously be cautious as it is based upon subgroup analyses which effectively break the randomization of the original study. Nonetheless, there is a clear message of practical clinical importance for the "best practice" treatment of angina: exclusive "reliance" on RAS blockade may be misplaced and greater attention should be directed towards control of BP (and particularly SBP) by means, if necessary, of combinations of BP-lowering drugs.

\section{ACKNOWLEDGMENTS}

Peter Meredith and Henry Elliott have received research grants, honoraria for consultancy, advisory board attendance and 
speaker fees from a number of pharmaceutical companies, including AstraZeneca, Bayer Schering Pharma, Boehringer Ingelheim, GSK, MSD, Pfizer, and Takeda. The analysis for this study was supported by an unrestricted grant from Bayer Schering Pharmaceuticals.

Open Access. This article is distributed under the terms of the Creative Commons Attribution Noncommercial License which permits any noncommercial use, distribution, and reproduction in any medium, provided the original author(s) and source are credited.

\section{REFERENCES}

1. Fox K, Garcia MA, Ardissino D, et al. Guidelines on the management of stable angina pectoris: executive summary: the Task Force on the Management of Stable Angina Pectoris of the European Society of Cardiology. Eur Heart J. 2006;27:1341-1381.

2. Yusuf S, Sleight P, Pogue J, Bosch J, Davies R, Dagenais G. Effects of an angiotensin-convertingenzyme inhibitor, ramipril, on cardiovascular events in high-risk patients. The Heart Outcomes Prevention Evaluation Study Investigators. N Engl J Med. 2000;342:145-153.

3. Fox KM. EURopean trial On reduction of cardiac events with Perindopril in stable coronary Artery disease Investigators. Efficacy of perindopril in reduction of cardiovascular events among patients with stable coronary artery disease: randomised, double-blind, placebo-controlled, multicentre trial (the EUROPA study). Lancet. 2003;362:782-788.
4. Braunwald E, Domanski MJ, Fowler SE, et al. Angiotensin-converting-enzyme inhibition in stable coronary artery disease. N Engl J Med. 2004; 351:2058-2068.

5. Poole-Wilson PA, Lubsen J, Kirwan BA, et al. Effect of long acting nifedipine on mortality and cardiovascular morbidity in patients with stable angina requiring treatment (ACTION trial): randomised controlled trial. Lancet. 2004;364:849857.

6. Lubsen J, Wagener G, Kirwan BA, et al. Effect of long-acting nifedipine on mortality and cardiovascular morbidity in patients with symptomatic stable angina and hypertension: the ACTION trial. J Hypertens. 2005;23:641-648.

7. Lubsen J, Poole-Wilson PA, Pocock SJ, et al. Design and current status of ACTION: a coronary disease trial investigating outcome with nifedipine GITS. Eur Heart J. 1998;19(suppl. I):I20-I32.

8. Elliott HL, Meredith PA. Preferential benefits of nifedipine GITS in systolic hypertension and in combination with RAS blockade: further analysis of the 'ACTION' database in patients with angina. J Hum Hypertens. 2010 [Epub ahead of print].

9. Lubsen J, Voko Z, Poole-Wilson PA, et al. BP reduction in stable angina by nifedipine was related to stroke and heart failure reduction but not to coronary interventions. J Clin Epidemiol. 2007;60:720-726.

10. Yusuf S, Teo K, Anderson C, et al. The Telmisartan Randomised AssessmeNt Study in ACE iNtolerant subjects with cardiovascular Disease (TRANSCEND) Investigators. Effects of the angiotensin- receptor blocker telmisartan on cardiovascular events in high-risk patients intolerant to angiotensin converting enzyme inhibitors: a randomised controlled trial. Lancet. 2008;372:1174-1183. 